\title{
INTESTINAL OBSTRUCTION IN THE NEWBORN INFANT*
}

\author{
BY \\ M. GROB \\ From the Surgical Department, the Children's Hospital, Zurich
}

The syndrome known as mechanical ileus or intestinal obstruction is a many-sided problem, the solution of which, in childhood and especially in the newborn infant, is one of the most difficult tasks of the paediatric surgeon, as regards both diagnosis and treatment.

The diagnostic difficulties are due above all to the fact that just at this age the pathological changes causing intestinal obstruction are so numerous. As intestinal obstruction is no more than a symptom, the physician diagnosing it is at most half way to recognition of the disease. The site and type of obstruction are not as yet clear.

The difficulties in treatment arise from the fact that ileus is not only a local affection but also a grave general disease in which quick decisions must be made.

\section{Clinical Features}

The clinical picture depends essentially on three factors: on the level of the obstruction, on its duration and on whether it is complete or partial. In high-seated obstruction vomiting is one of the first signs and abdominal distension is limited to the epigastrium. In low-seated obstruction distension of the whole abdomen precedes vomiting. In the newborn infant swallowed air reaches the rectum only after 12-24 hours. Therefore if the obstruction is at a low level, abdominal distension will rarely be present before 12 hours have elapsed. On the other hand, it should be remembered that meteorism during the first 12-36 hours of life may be physiological. In partial obstruction, such as congenital stenosis of the intestine, vomiting is the first and most striking symptom, while abdominal distension may be minimal because the intestinal air is able to pass through the stenosis.

Investigation of bowel function in the first days of life, that is to say the presence or absence of meconium, may provide important clues in the diagnosis of intestinal obstruction, although no hard

* A paper read at a meeting of the British Association of Paediatric Surgeons held in Liverpool in June, 1959. and fast rules exist in this respect. Delayed passage of meconium should always lead one to suspect low intestinal obstruction, such as atresia of the rectum or colon, meconium ileus or Hirschsprung's disease, although this symptom may also occur in normal babies. On the other hand, evacuation of meconium does not exclude a primary high-seated intestinal obstruction, since meconium is produced in all parts of the intestinal tract and bile pigments reach the intestine by way of the blood.

Microscopical examination of the meconium for cornified epithelial cells and lanugo hairs makes it possible to distinguish between cases with early primary obstruction of the intestine and those in which the obstruction developed later in foetal life by strangulation, volvulus or intussusception. In primary congenital atresia of the bowel these meconium elements are absent.

\section{Radiology}

Radiological findings are of prime importance in diagnosis. In every case of ileus in the newborn infant radiological examination in the upright position is called for. Plain films are important since conclusions may be reached as to the site of the obstruction from the number and position of distended loops and fluid levels. In duodenal obstruction the radiological picture is typical. It shows a dilatation of the stomach and only one pathological fluid level on the right of the spine (Fig. 1). The corresponding finding at operation in such a case is seen in Fig. 2. The superior segment of the duodenum is greatly distended and as large as the stomach. In lower intestinal obstruction a plain film in the upright position reveals multiple distended loops and fluid levels (Fig. 3).

Furthermore, an enema with a contrast medium should in no case be omitted, as in newborn babies it is often impossible to decide whether the distended loops belong to the small bowel or to the colon. Such a procedure also provides valuable information as to the permeability and calibre of the colon and the site of the proximal colon. It may thus reveal 


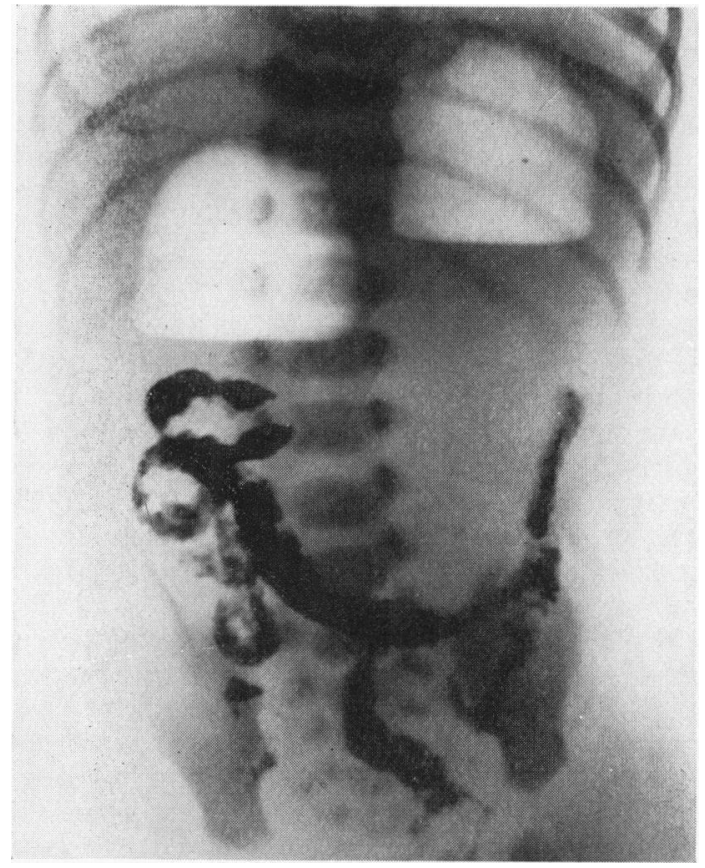

FIG. 1.-Duodenal atresia in a 5-day-old infant.

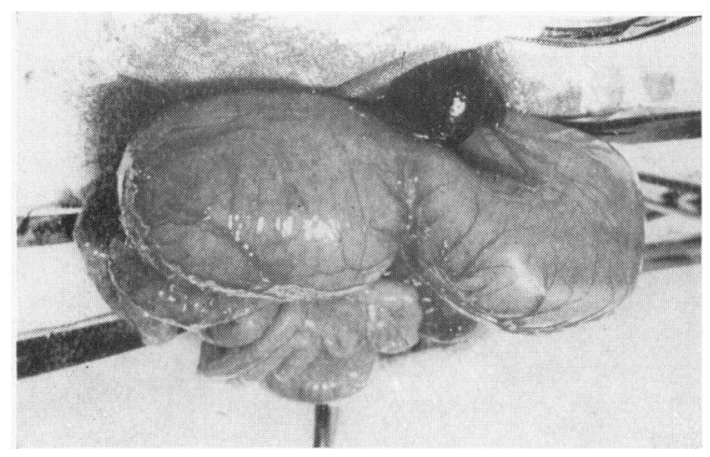

FIG. 2.-Operative findings in duodenal atresia. Greatly dilated superior segment of duodenum.

the presence of atresia, Hirschsprung's disease or malrotation.

Barium is generally used for contrast enema, but the use of resorbable preparations, such as Ioduron or Triopac, is advisable at least in cases of suspected perforations.

Oral administration of barium should be avoided as it may change the patient's condition for the worse and thereby complicate operation. It is, moreover, dangerous if aspiration occurs. A further

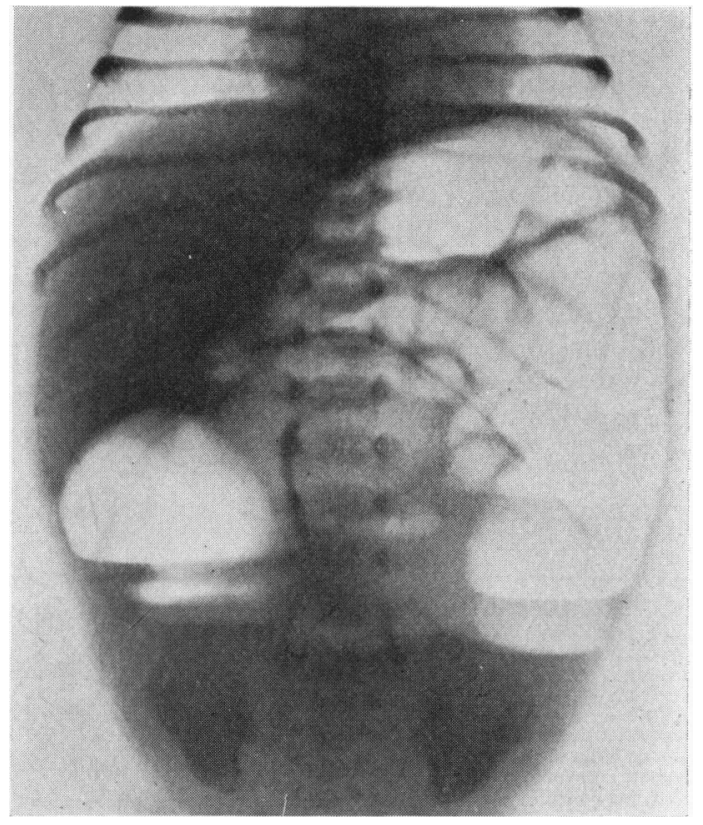

FIG. 3.-Multiple distended loops and fluid levels in lower intestinal obstruction.

disadvantage of oral administration as compared with an enema is loss of time. Long radiological procedures may mean that the right moment for operation is missed. It is valueless to endanger the child's life by long drawn out diagnostic procedures, since laparotomy is indicated in any case, at which diagnosis can be completed.

\section{Preliminary Treatment}

Apart from local symptoms, intestinal obstruction results in disturbances of the general condition.

Loss of fluid due to vomiting and reduced resorption from the bowel produce dehydration and disturbances in electrolyte balance, such as hypochloraemic alkalosis and hypokalaemia. Intraintestinal and intraperitoneal transudation produce hypoproteinaemia by loss of plasma. Respiratory difficulties owing to abdominal distension may result in anoxaemia and cyanosis.

Treatment must first of all aim at combating these general disturbances. It would be a grave mistake to expose the baby to the danger of operative shock without adequate preliminary treatment. This consists of the supply of fluid, electrolytes, blood and plasma by intravenous infusion, and employment of a gastro-intestinal suction system. The decrease of abdominal distension by means of a 
naso-gastric tube facilitates operative intervention and diminishes operative shock. Intestinal suction is not only of great use in pre-operative treatment, but also during post-operative care, since it prevents paralytic ileus.

\section{Types of Intestinal Obstruction}

The most frequent disorders that the surgeon has to keep in mind are summarized in Table 1. It is clear from this Table that as a rule we have to deal with congenital malformations.

TABLE 1

CAUSES OF INTESTINAL OBSTRUCTION IN THE NEWBORN INFANT

Atresia and stenosis: duodenum, jejunum, ileum, colon, rectum, anus

Annular pancreas
Meconium ileus

Disturbances of foetal intestinal rotation: nonrotation, malrotation, common mesentery

Peritoneal bands

Meckel's diverticulum, omphalo-mesenteric duct

Enterogenous cysts (duplication)

Mesenteric cysts

Mesenteric cysts
Intestinal polyps

Intussusception

Megacolon (Hirschsprung's disease)

Strangulated inguinal hernia

Strangulated diaphragmatic hernia

Peritonitis

Firstly, the malformation may constitute the immediate cause of the obstruction, as in congenital atresia, annular pancreas, peritoneal bands, etc., or secondly, it may only be a predisposing factor, for instance, in cases of common mesentery, persistent omphalo-mesenteric duct or in diaphragmatic hernia. Thirdly, we have congenital defects, predominantly functional in type, such as meconium ileus and Hirschsprung's disease.

Atresia and Stenosis. In congenital atresia and stenosis of the small intestine, or more rarely of the colon, we have to distinguish between primary and secondary forms.

Primary atresia and stenosis is due to a persistence of epithelial proliferation existing during the fifth to twelfth weeks of foetal life and occluding temporarily the lumen of the intestinal tract. Remaining portions of these epithelial masses may persist as one or several mucosal septa with or without a central opening which obstruct the lumen. Primary atresia and stenosis manifest themselves only by a difference in calibre. No interruption in intestinal continuity can be noticed from the outside (Figs. 4-7).

On the other hand, congenital atresia may also be due to a pathological process during the foetal period which causes secondary obstruction of the bowel. Pathological processes, such as foetal volvulus, intussusception, incarcerated hernia and meconium ileus, may cause local necrosis of an intestinal segment, thereby producing atresia. These secondary atresias, which are commonly situated at the level of the ileum, are characterized by a gap between the bowel ends or a cord which usually has no mucosal lining. Cicatrization at the ends of the atretic segments and defects in the mesentery are frequent findings. Fibrous nodules on the serous coat and areas containing calcified debris are the remnants of former perforation.

We have observed a case of intestinal atresia in which the collapsed distal stump still contained the remains of an invaginated segment. In another case an incarceration of the lower ileum in an omphalocoele produced a secondary atresia (Figs. 8 and 9).

While in former times operative treatment of atresia or stenosis was rarely successful, modern operative technique and especially adequate postoperative care have considerably improved the prognosis. As a rule the surgeon will try to overcome the obstruction by entero-anastomosis, the method of choice being end-to-end anastomosis as an initial procedure. Enterostomy as a palliative procedure should be considered only in cases in which the obstruction is situated in the terminal ileum or more distal to it. Enterostomy at a higher level is always fatal because of the excessive loss of fluid.

In the performance of entero-anastomosis the difference in size of the pre- and post-stenotic segment may cause some difficulty. In this situation the decompression of the proximal dilated intestine by puncture, suction of direct opening and the injection of normal salt solution into the collapsed narrow distal segment may overcome the discrepancy in size.

In cases of duodeno-jejunostomy for duodenal stenosis or atresia, the passage of a transanastomotic feeding tube with its top lying in the contracted jejunum is advisable because it allows early feeding and dilatation of the distal contracted loops.

But even in correctly performed anastomosis dilatation of the proximal segment with failure of propulsive activity may persist. Resection of the dilated proximal bulb before end-to-end anastomosis seems therefore to be indicated, especially in cases of secondary atresia with cicatrization of the stumps.

Meconium Ileus. A special kind of intestinal obstruction in the newborn infant occurs in meconium ileus, which requires medical as well as 


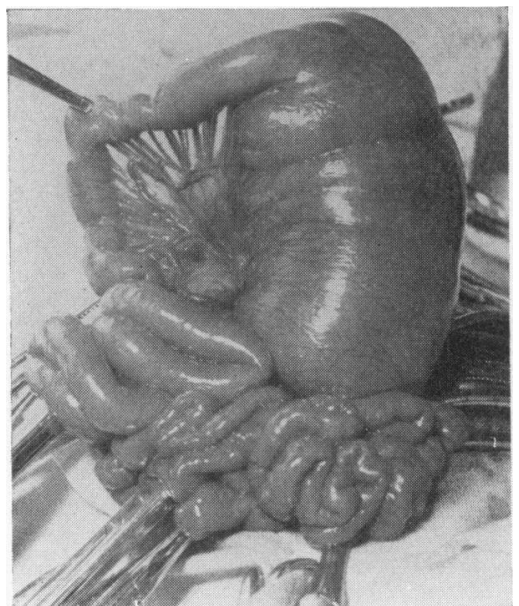

FIG. 4.-Primary stenosis of jejunum.

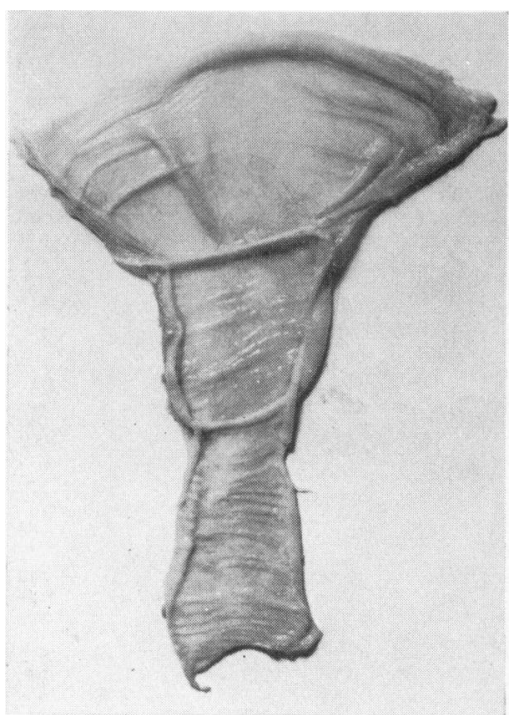

FIG. 6.-Removed and opened specimen (case shown in Fig. 5) with two mucosal folds which formed the stenosis.

surgical treatment. Meconium ileus is a typical example of intraluminal obstruction, which is caused by an abnormal inspissation of the meconium, especially in the lower segments of the ileum. The abnormal consistency of the meconium is due not only to a deficiency of pancreatic enzymes as a consequence of the co-existing fibrocystic disease of the pancreas; it is rather the expression of a generalized disease of the secreting glands, which is known as mucoviscidosis. The mucous glands of

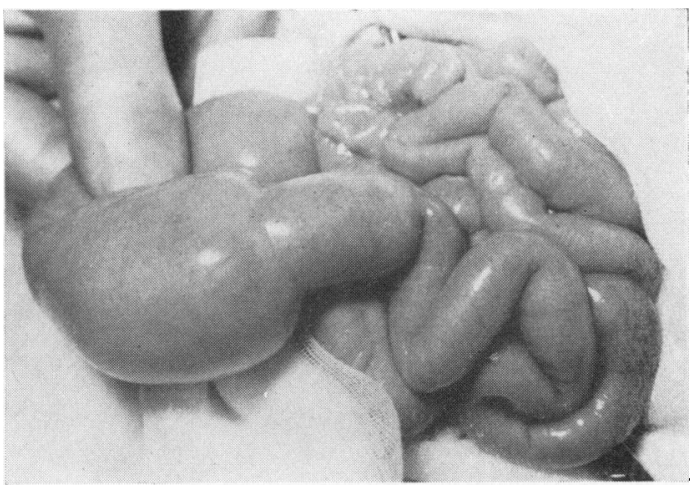

FIG. 5.-Primary double stenosis of jejunum.

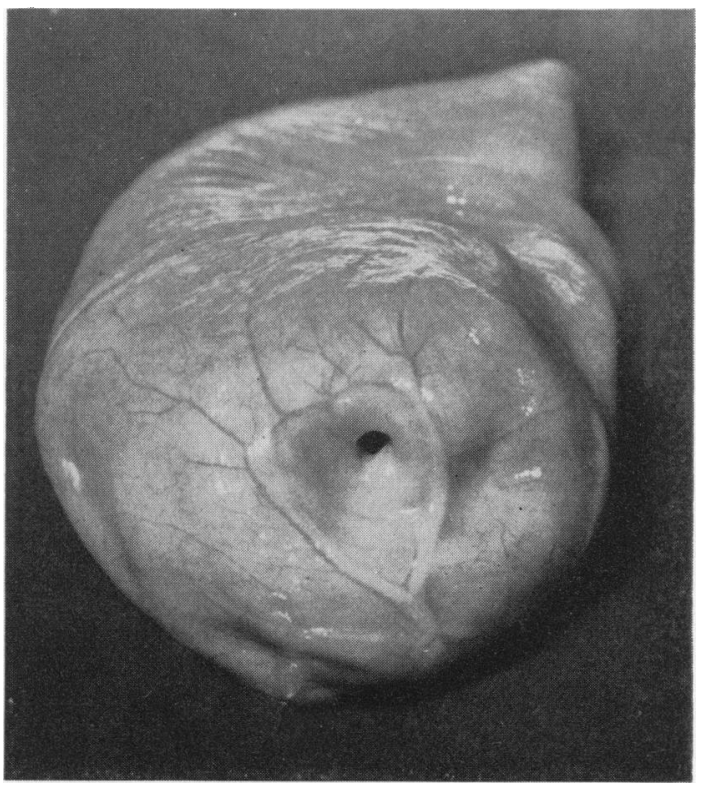

FIG. 7.-Small opening in septum causing stenosis (case shown in Fig. 5).

the bowel, too, produce an exceedingly sticky secretion which is firmly attached to the inspissated meconium.

Operation reveals dilated jejunal loops filled with gas and thick meconium, the ileum appearing like a string of beads due to greyish, putty-like knots. The colon is generally empty (Fig. 10).

The diagnosis is already evident from the radiograph (Fig. 11). The dilated upper loops of the intestine do not present fluid levels in the picture in 


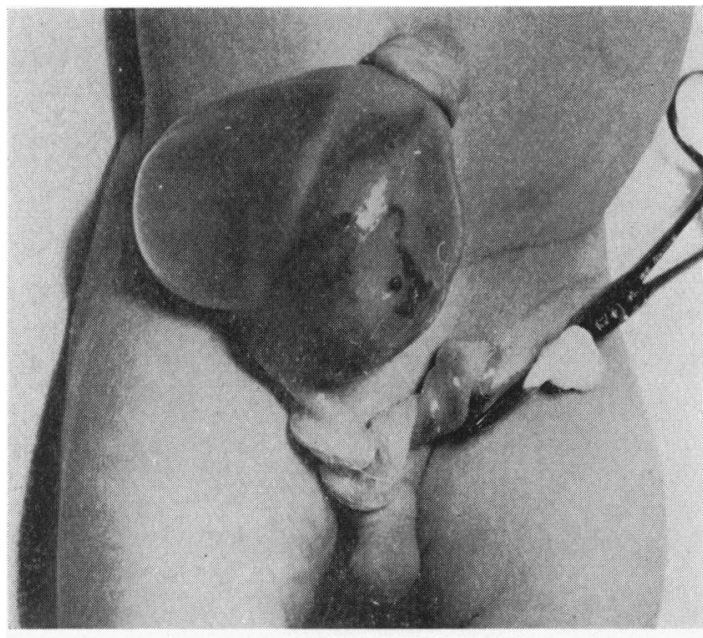

FiG 8

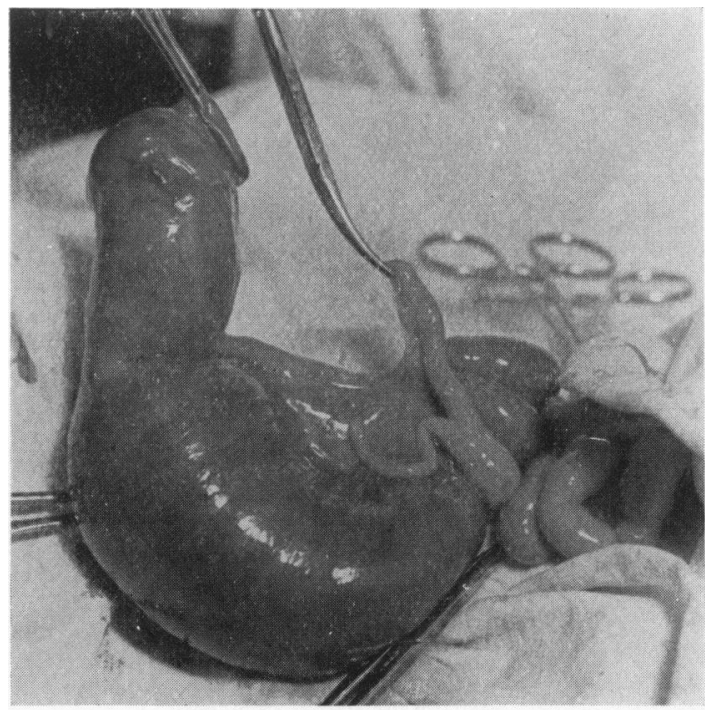

FIG. 9.

FIG. 8.-Omphalocoele in a newborn baby with a very narrow umbilical ring and incarcerated transparent mass.

Fig. 9.-Finding at operation in case shown in Fig. 8: secondary atresia of lower ileum owing to strangulation by narrow umbilical ring during foetal period.

FIG. 10.-Typical appearance of meconium ileus.

FIG. 11.-Meconium ileus with several dilated loops of different size without fluid levels. Mottled meconium masses on right side.

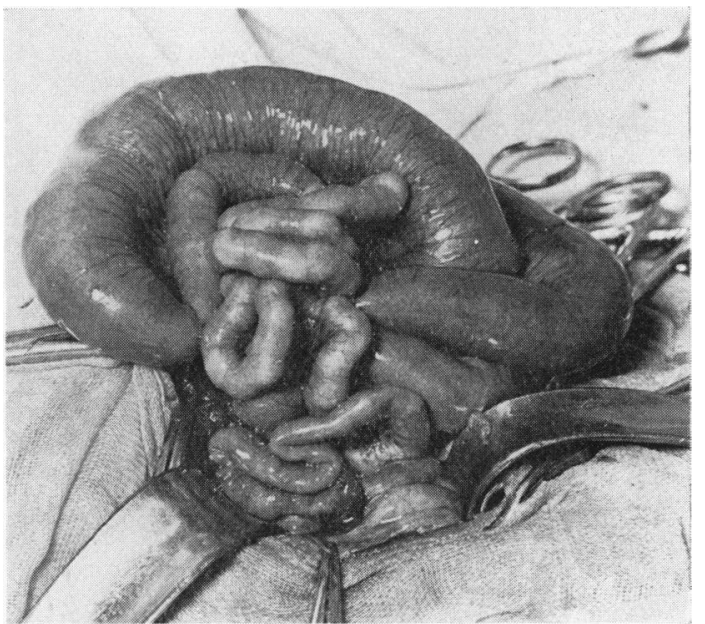

FIG. 10 .

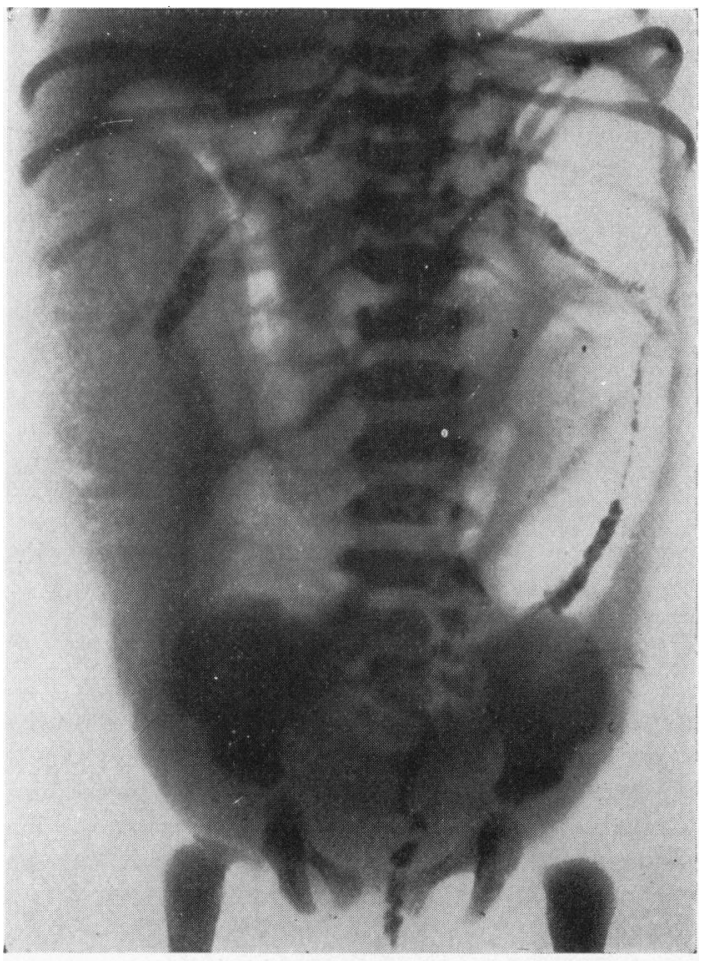

FIG. 11. 


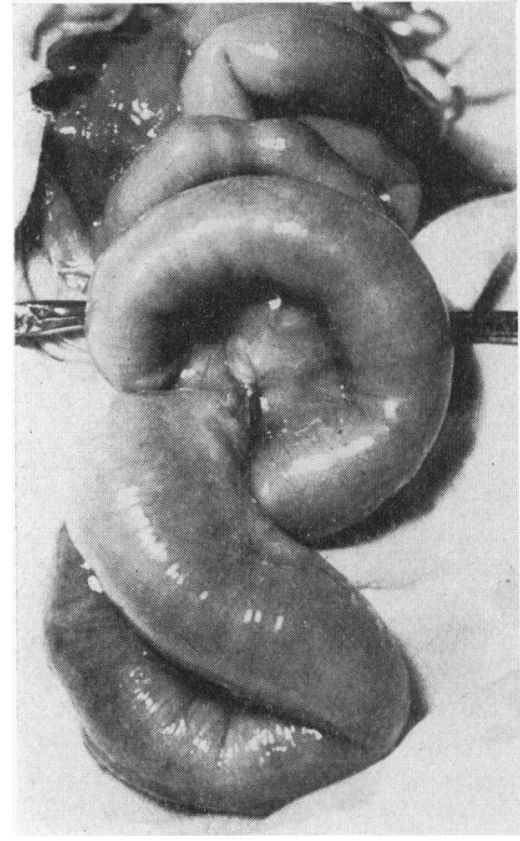

FIG. 12.-Meconium ileus with volvulus.

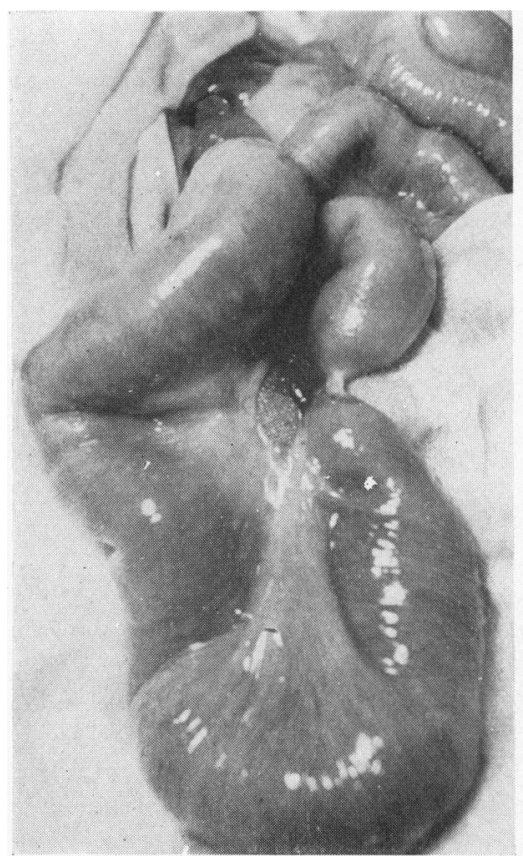

FIG. 13.-(Case shown in Fig. 12.) Reduction of volvulus reveals a completely squashed loop (secondary atresia). the upright position because the thick gluey meconium adheres to the intestinal wall. The meconium masses manifest themselves by granular spots. The mottled appearance is due to infiltration of the meconium by small air bubbles.

An enema with a contrast medium shows the colon, which is free of meconium and has the appearance of a so-called 'microcolon'.

Meconium ileus may produce several complications even during the foetal period. The accumulated meconium masses may cause ischaemia, necrosis and perforation of the intestinal wall, thus producing aseptic meconium peritonitis, which may cause adhesions and strangulations. These cases present typical calcified granulations in the peritoneal cavity corresponding to foreign body reactions.

Not infrequently the enlarged and heavy loops of the upper intestine become twisted upon themselves and give rise to volvulus formation with or without secondary atresia of the strangulated segments. Fig. 12 represents a volvulus of this origin. Reduction of the volvulus reveals a completely squashed loop (Fig. 13). In this way secondary atresias are formed. In the case of Fig. 14 the same process took place at an early stage of foetal life. The result is a complete interruption of the continuity of the bowel.

In the treatment of meconium ileus the following facts are of crucial importance. We know that it is impossible to estimate the degree of severity by clinical and radiological investigations, or to distinguish uncomplicated from complicated cases with volvulus, perforations and secondary atresias. Laparotomy is therefore indicated in any case.

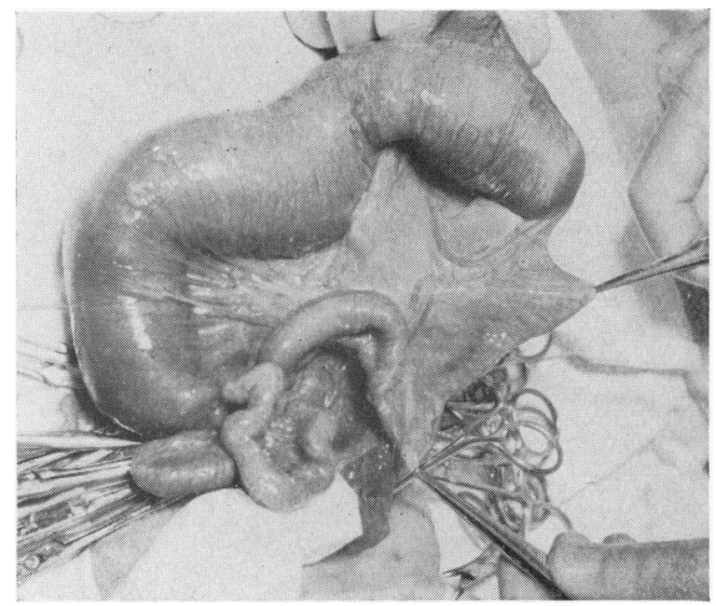

Fig. 14.-Meconium ileus with secondary atresia of lower ileum caused by volvulus at an early foetal stage. 
In uncomplicated cases the first thing is to relieve the increasing and dangerous intestinal distension by suction. The removal of the obstacle, the sticky thick meconium masses in the ileum, cannot possibly be performed by mechanical procedures. On the other hand, it has been proved that after administration of pancreatic enzymes the meconium may be expelled spontaneously in a few days. Therefore we make a small incision in the lowest portion of the distended intestine, deflate the upper loops and introduce a powdered preparation of pancreatic enzymes into the intestinal lumen. Before we close the abdominal wound a tube is passed through the nose into the stomach and duodenum under direct vision. In this way the intestine can be deflated during subsequent days and further proteolytic enzymes can be instilled through the tube.

While this simple treatment is successful in uncomplicated cases, more severe forms require enterostomy, through which enzyme instillations can be administered. Although results as regards relief of the obstruction seem at first glance to be satisfactory, long-term results are disappointing. Most of these babies die sooner or later of overwhelming pulmonary infection in spite of adequate diet, administration of pancreatic enzymes and antibiotic treatment.

Abnormal Bowel Positions. Other not infrequent conditions causing intestinal obstruction in the newborn infant are abnormal positions of the bowel

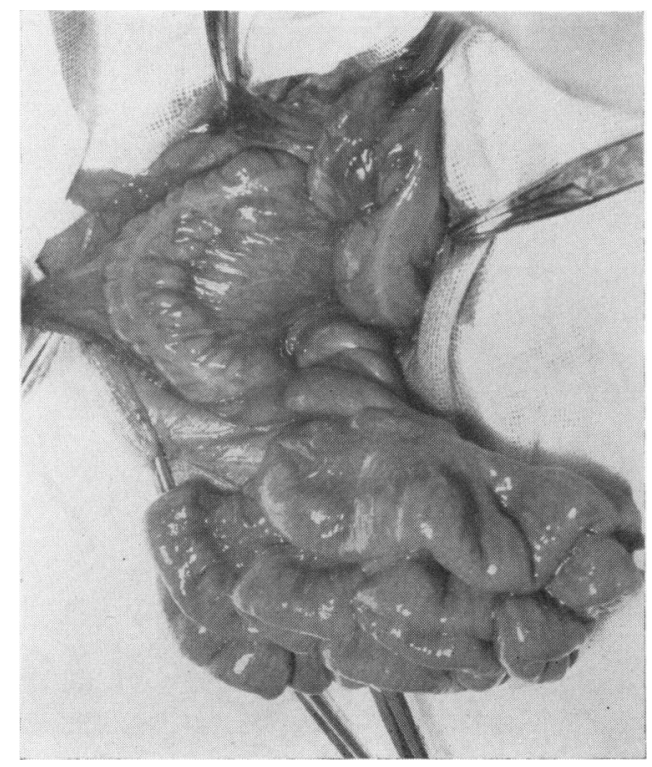

Fig. 15.-Volvulus in a newborn infant with common mesentery.

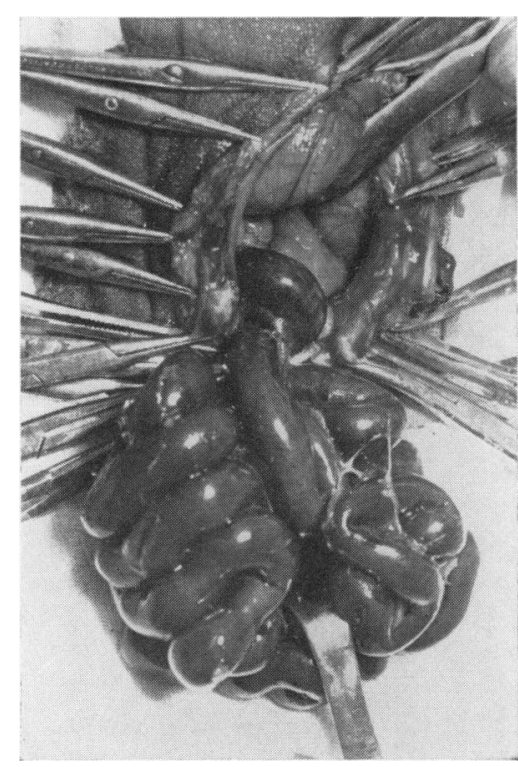

FIG. 16.-Infarction of intestine in a newborn infant owing to common mesentery with volvulus.

due to lack of normal rotation of the midgut during the foetal period. The recognition of such conditions often demands considerable spatial imagination on the part of the surgeon.

The normal position of the bowel is reached by a rotation of the midgut of $270^{\circ}$ in an anti-clockwise direction. The midgut represents that portion of the alimentary tract from the duodenum to the middle of the transverse colon, which is supplied by the superior mesenteric artery. To reach a normal position, the mesentery of the ascending colon has to become fixed to the right posterior abdominal wall.

The rotation may be incomplete, it may stop after $90^{\circ}$ or $180^{\circ}$ or take place in a reverse direction. In addition, failure of attachment of the mesentery along the right lateral and posterior abdominal wall is nearly always present, but this anomaly, so-called common mesentery, may also be encountered in cases with normal rotation.

The common mesentery can obviously give rise to twisting of the entire mass of the small intestine and colon around the small mesenteric root, which generally takes place in a clockwise direction (Fig. 15). The twisted mesenteric pedicle may strangulate the inferior part of the duodenum and produce symptoms of duodenal stenosis. In rare cases, in which the twisted mesenteric root strangulates the mesenteric vessels, infarction of the entire intestinal mass may ensue (Fig. 16). 
Another anomaly causing obstruction of the duodenum results from compression of the lower duodenum by an overlying caecum or ascending colon. This condition may be encountered in cases with incomplete or reversed rotation, in which the caecum is placed in the duodenal area and becomes fused with the posterior abdominal wall.

A third form of obstruction may be seen in cases with reversed rotation when the duodenum reaches its final position in front of, and the transverse colon behind, the mesenteric root. This condition, known as retroposition of the transverse colon, may produce an obstruction of the proximal colon, especially if it is complicated by an additional volvulus. Visualization of the colon by radiography with contrasting barium almost always supplies significant information. When the caecum and ascending colon are displaced to the left and upper part of the abdomen a volvulus of the midgut should always be suspected (Figs. 17 and 18).

Even so-called internal hernia, or mesocolic hernia, may be due partly to anomalous intestinal rotation. The tendency of the proximal colon to reach its normal position on the right, independently of previous foetal rotation, may in cases of non-

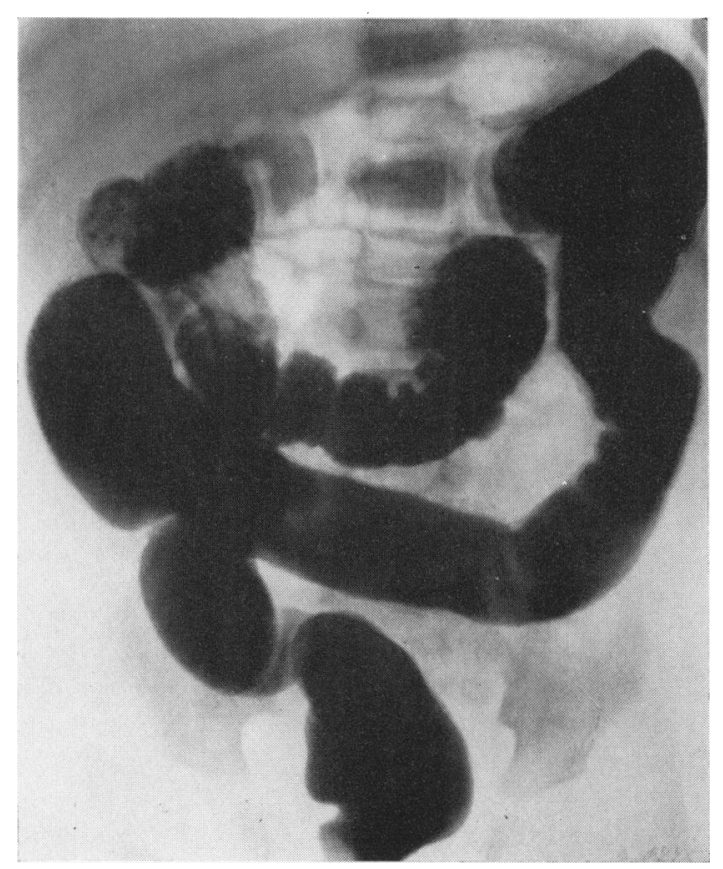

FIG. 17.-Retroposition of transverse colon (reversed rotation) with additional volvulus. Caecum lies in an anomalous position in left upper side of abdomen. Filling defect of transverse colon due to compression by twisted mesenteric pedicle. rotation or reversed rotation lead to partial or total envelopment of the small intestine by the ascending mesocolon. Thus internal hernia is produced in which intestinal loops may be incarcerated (Fig. 19).

Operative treatment of the abnormal position and rotation should not only comprise the reduction of a volvulus or the severance of strangulating bands, but the ascending colon should be fixed to the right posterior abdominal wall in a normal position. Our own experience with mesenteric vessels has taught us that fixation at the right site is absolutely essential in order to obtain optimal blood supply.

Enterogenous Cyst. Another congenital malformation which has to be taken into account in cases of intestinal obstruction is the enterogenous cyst, or duplication of the bowel, which is seen in about $20 \%$ of cases even in the neonatal period.

These structures, which may be tubular, cystic or diverticular, can branch out from the entire bowel, but they usually lie in the region of the lower ileum and caecum. In spite of this, they have embryologically nothing to do with Meckel's diverticulum. The duplications are due to disturbances during an early foetal period in which

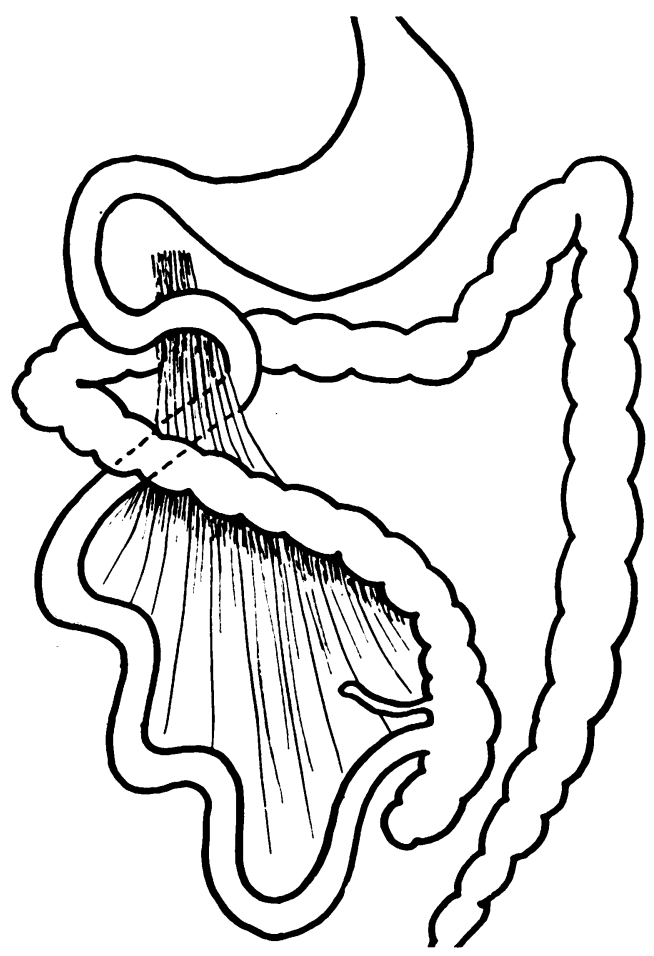

Fig. 18.-Diagram of case shown in Fig. 17. 


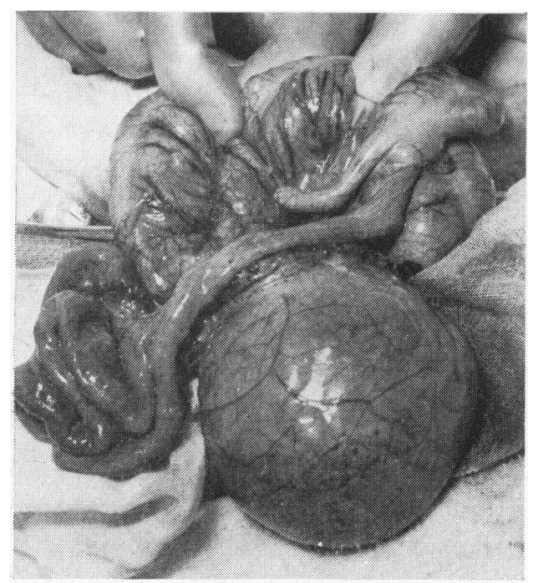

FIG. 19.

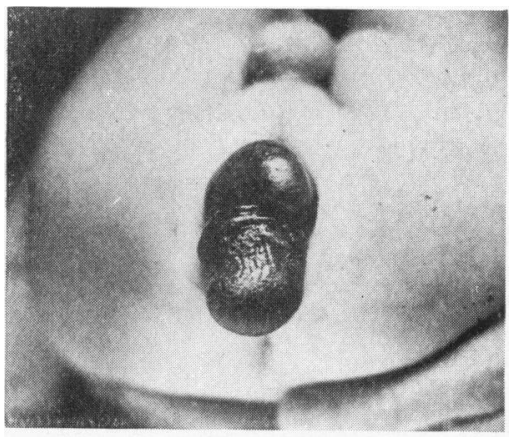

FIG. 20.

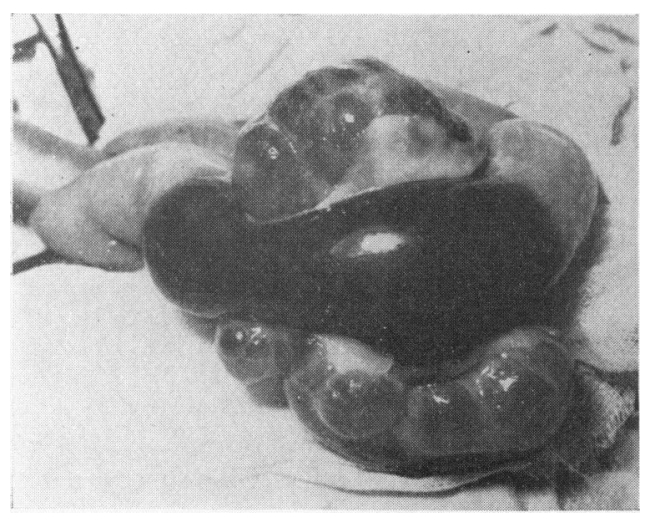

FIG. 22.
FIG. 19.-Internal hernia containing several incarcerated loops. Hernial sac is formed by mesentery of ascending colon.

FIG. 20.-Intussusception in a newborn baby with intussusceptum protruding from anus owing to ileal duplication.

FIG. 21.-Resected ileo-caecal specimen from case shown in Fig. 20. Note enterogenous cyst in lower ileum.

FIG. 22.-Multilocular lymphatic mesenteric cyst producing intestinal obstruction and stasis of adjacent bowel segment.

Fig. 23.-Hour-glass-shaped chylous mesenteric cyst which contained large quantity of yellow, milky fluid.

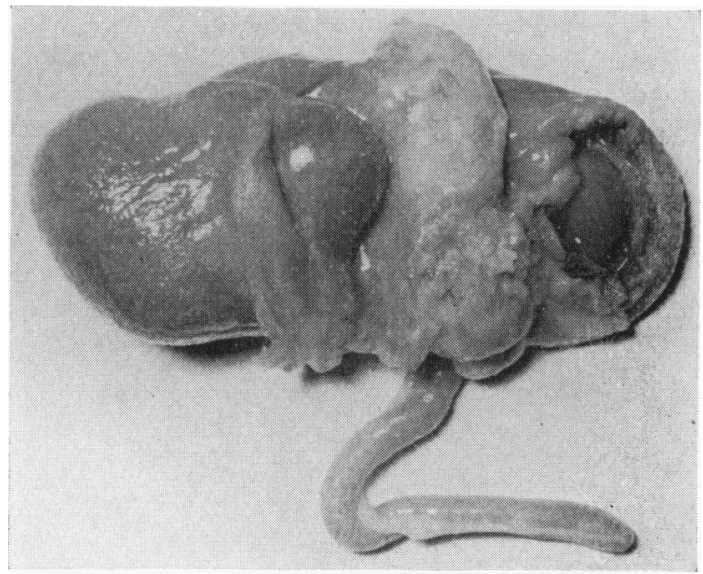

FIG. 21.

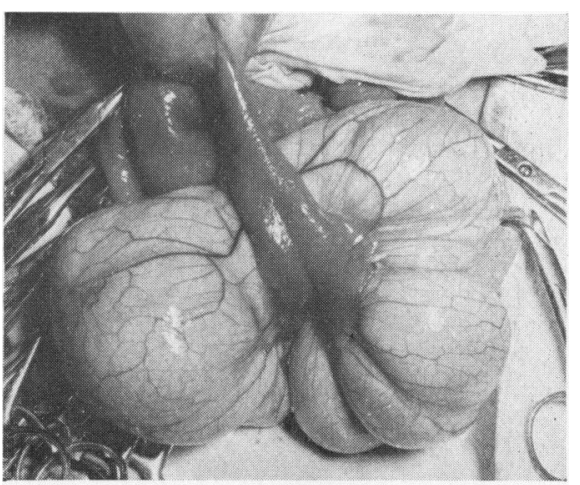

FIG. 23. 
the chorda dorsalis is separated from the entoderm.

These cystic structures, closely attached to some portion of the alimentary tube, grow continuously in size by retention of secreted mucosal fluid, thereby causing obstruction of the intestinal lumen.

Since these duplications are supplied with blood by the same vessels as is the adjacent bowel, it is impossible to resect the cyst without impairment of the blood supply to the contiguous intestinal segment. Therefore the segment in question has also to be resected.

Cysts of this type may not only produce intestinal obstruction, but they may also act as a leading point in the development of intussusception. Fig. 20 shows a newborn baby with intussusception, the tip of the intussusceptum protruding from the anus. It contained a single cystic tumour. Surgical reduction of the intussusception presented no difficulty. After reduction, the cystic tumour proved to be a duplication of the lower ileum which had passed the whole colon within a few hours. Ileo-caecal resection was performed (Fig. 21) and the post-operative course was uneventful.

Mesenteric Cyst. Other structures, which have to be differentiated from enterogenous cysts, are lymphatic mesenteric cysts. These tumours rarely cause intestinal obstruction in the newborn infant. The cysts are thin-walled and multilocular and often have the shape of an hour-glass. They contain serous, colourless or chylous yellowish fluid (Figs. 22 and 23). Smaller cysts can be dissected out from the mesentery, while large ones require intestinal resection and end-to-end anastomosis.

Hirschsprung's Disease. Lastly I should like to make brief mention of Hirschsprung's disease or aganglionic megacolon.

Newborn babies with this disease almost always show difficulty in evacuation of meconium, constipation, vomiting and abdominal distension, all symptoms of intestinal obstruction which, however, vary in intensity.

Often an obstruction of the small bowel is suspected, particularly as plain radiography shows dilated loops with fluid levels. To exclude this a contrast enema is absolutely essential. It shows that the dilatation is situated in the colon. The picture typical for Hirschsprung's disease with contracted rectosigmoid is, however, frequently not so marked in the newborn baby as it is some weeks later. In case of doubt a biopsy from the rectal musculature should be performed. Microscopical examination will prove whether ganglion cells are present or not.
One rare though severe complication of Hirschsprung's disease, which may occur in the first days of life, is perforation of the distended colon (Fig. 24). We have observed it in three cases: twice in the region of the left colonic flexure and once in the caecum. In all three cases colostomy was performed at the site of perforation with good results.

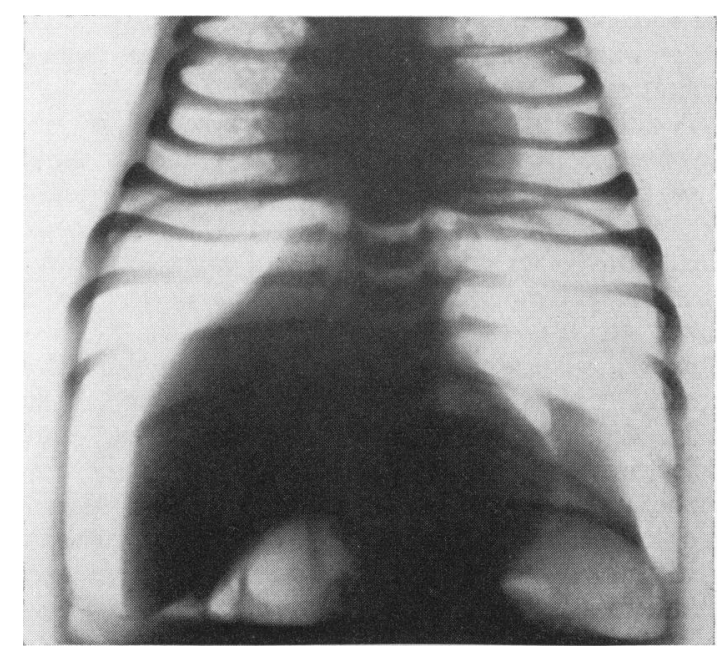

FIG. 24.-Pneumoperitoneum after perforation of colon in a 2-day-old baby suffering from Hirschsprung's disease.

In the treatment of megacolon in the newborn baby conservative measures will, as a rule, be resorted to at the start with frequent enemas to combat the gaseous distension. In most cases this will prove successful for some time. Should difficulties arise in controlling the distension, temporary colostomy is advisable. Nevertheless, we do not hesitate in such cases to perform rectosigmoidectomy by Duhamel's method even in the first few months of life. This retrorectal pull-through is technically simple, as the rectum has to be mobilized only on its dorsal side, which is easy to accomplish. The intervention is therefore relatively short and produces no shock. It prevents secondary strictures at the site of the anastomosis and disturbances of bladder function.

We have employed this method in 15 cases, six of whom were infants aged from 6 weeks to 8 months. Results were very satisfactory regarding healing of the megacolon, but later investigation of these children showed that they suffered from numerous evacuations, some even from actual diarrhoea, as a result of the destruction of the internal sphincter. 
We therefore advise the following modification of Duhamel's procedure: we incise the posterior wall of the rectum $1.5 \mathrm{~cm}$. above the internal sphincter, thus conserving this important muscle. The proximal stump of the colon is pulled through this incision and not through one at the level of the anus. Details of this special technique will be published shortly. The apparent level of the transitional zone in young infants may be misleading; biopsy of the colon muscle tissue during operation is therefore advisable.

A difficult problem in the newborn period, which, however, arises only seldom, is presented by those cases in which the aganglionic zone extends throughout the whole colon as far as the lower ileum. Deflation by an enema is in these cases ineffective and the clinical picture corresponds to that of a lower stenosis of the small bowel. We recently observed such a case, which at operation showed a greatly dilated and hypertrophic lower ileum (Fig. 25). Ileostomy was performed and total colectomy was provided for at a later date. Unfortunately the infant died of peritonitis. Microscopical examination revealed that the hypertrophic ileal loops contained ganglion cells in normal numbers whereas the terminal part of the ileum contained only a few ganglion cells, and throughout the caecum and colon they were completely absent. The peculiar thing about this case was that the child showed no symptoms of intestinal obstruction as long as she was breast-fed. Only after changing to artificial feeding did such symptoms appear.

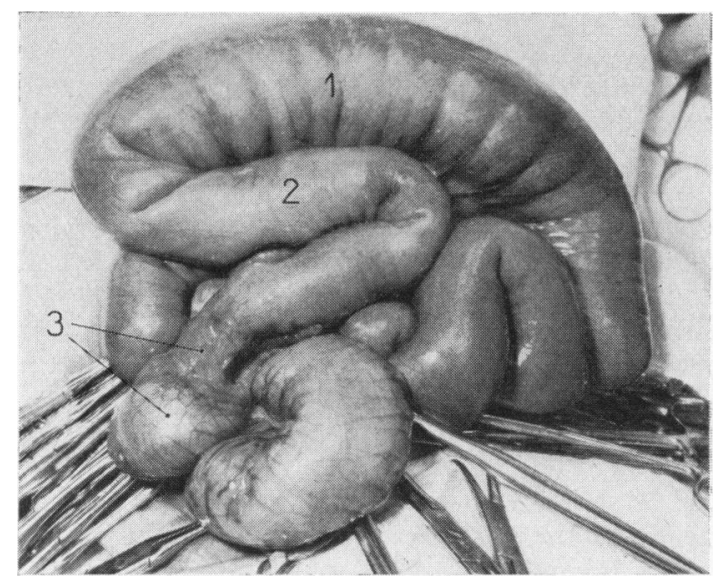

FIG. 25.-Hirschsprung's disease with aganglionic zone throughout colon and terminal ileum: $1=$ hypertrophic and dilated ileum with normal number of ganglion cells; $2=$ only few ganglion cells ; 3 = ganglion cells completely absent.

In conclusion I should like to emphasize that I am fully aware of the incompleteness of my remarks concerning intestinal obstruction in the newborn baby. Many problems could only be touched upon.

Intestinal obstruction in a newborn baby is always a delicate and critical matter demanding rapid decisions. Success in these cases depends not only on early diagnosis and appropriate operation, but also upon the quality of pre- and post-operative care, of suitable anaesthesia, and, last but not least, upon the constant and untiring efforts of our nurses. 